\title{
ARTICLE
}

\section{Development of NaI Survey Protocol for Induced Radioactive Materials on Clearance}

\author{
Hirokuni YAMANISHI ${ }^{1 *}$, Amanda SABOUROV ${ }^{2}$, Murat KERIMBAEV ${ }^{2}$, and James C. LIU ${ }^{2}$ \\ ${ }^{I}$ National Institute for Fusion Science, 322-6, Oroshi-cho, Toki, Gifu 509-5292, Japan \\ ${ }^{2}$ SLAC National Accelerator Laboratory, 2575, Sand Hill Road, Menlo Park, CA 94025, USA
}

\begin{abstract}
A radiation survey with a survey meter is one of the processes required to declare a component non-radioactive for unrestrictive release. The gamma-rays which can reach the NaI detector were limited by geometry and self-shielding in the material. The region in which gamma-rays are detectable was examined by means of the MCNP code. The detector sensitivity to a Co-60 volume source in iron was obtained as $4378 \mathrm{cpm} /(\mathrm{Bq} / \mathrm{g})$ from the detectable region for uniform activity distribution. The minimum detectable activity (MDA) was defined as 4 times of the standard deviation of background count rate. The MDA was found to be lower than $0.1 \mathrm{~Bq} / \mathrm{g}$ which corresponds to $1 / 10$ of the ANSI-recommended screening level for Co-60.
\end{abstract}

KEYWORDS: NaI survey meter, gamma-ray, clearance level, minimum detectable activity

\section{Introduction}

The SLAC BaBar detector has been in operation for the study of CP violation for over a decade. Now that the program has been completed, the detector will be decommissioned and dismantled. The SLAC BaBar detector dismantling will generate a large quantity of metal-containing material. In the interest of beneficial reuse/recycling as well as to alleviate storage needs and disposal costs, it is desirable to release the BaBar components containing metals for recycling or reuse off site.

A radiation survey of the materials is one of the processes required to declare a BaBar component non-radioactive for unrestrictive release. One of the survey protocols is to conduct in-field gross beta-gamma surveys over all surfaces of the materials using a $\mathrm{NaI}(\mathrm{Tl})$ survey meter. The survey meter used in this report was the Ludlum Model 18 with Model 44-2 1-inch NaI detector. ${ }^{1-2)}$ The ANSI standard of the screening level for clearance is provided as a specific activity in $\mathrm{pCi} / \mathrm{g}$. ${ }^{3)}$ Therefore, we have to know the relationship between count rate and specific activity when a survey is conducted to a material by means of a $\mathrm{NaI}(\mathrm{Tl})$ detector. The minimum detectable activity (MDA) of a detector depends on the material and the gamma-ray energy as a radioactive source. When the object to be surveyed is large, the radiation that can be detected is limited because of self-shielding within the object. Therefore, the region in which radiation is detectable in the material needs to be known.

In this study, the detectable region and the MDA were obtained by Monte Carlo simulation using the MCNP code. ${ }^{4)}$ The calculations were conducted and the MDA of the instrument was examined as a sample case under the following conditions: for a 2-m diameter and 20-cm thickness iron block, for the isotope Co-60. Finally, the MDA was compared with $1 / 10$ of the screening level for

*Corresponding Author, E-mail:yamanisi@nifs.ac.jp

(C) Atomic Energy Society of Japan clearance.

If the activity has a distribution profile, the count rate will be changed even when the total activity is the same. Several types of activity profile were assumed as a function of depth and lateral distribution inside a volumetric component. Under these activity profiles, the expected count rate from the detectable region was compared with each other. And the effect of the activity distribution to the MDA was discussed.

\section{MCNP Calculation \\ 1. Instrument}

The geometry of the detector in MCNP was made based on the manual for the Ludlum NaI(Tl) detector Model 44-2. ${ }^{1)}$ The detector has a crystal size that is 1-inch in diameter and 1-inch in height. The detector probe has a 6-mm lead collar to reduce the background count rate. Pulse height tally is used in this calculation. It shows the energy deposited in the NaI crystal due to an incident gamma-ray.

\section{2. $R-Z$ model}

The cylindrical R-Z model was adopted as the basic geometrical structure for this MCNP calculation. The material to be surveyed was defined as an iron cylinder with 2-m diameter and 20-cm thickness. The detector was placed at $1 \mathrm{~cm}$ from the surface of the material on the central axis of the cylinder. Co-60 was chosen as the radioactive isotope activating the material.

The total region of the source extended from $10 \mathrm{~cm}$ below the surface and $40 \mathrm{~cm}$ radially from the central axis. The total region was divided into 5 layers in depth and 10 sectors in radius. As a result, one volume source of Co-60 - named as one source cell - has the shape of a cylinder or a cylindrical shell and has $2-\mathrm{cm}$ thickness and $4-\mathrm{cm}$ shell width on axial symmetry. Each source cell has a uniform distribution of the Co-60 radioactive source. Figure 1 shows the unit source cells on R-Z model. 


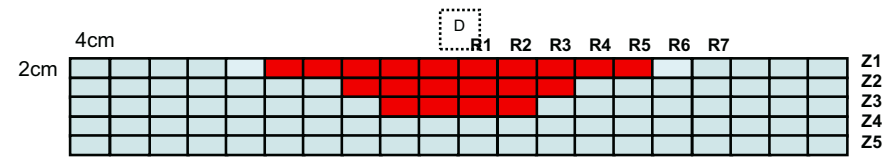

Fig.1 Cross section view of the arrangement of the unit source cells and detectable region

The pulse height due to each source cell was calculated individually. The data from each source cell is obtained by summing up pulse height data from $0.01 \mathrm{MeV}$ to $1.4 \mathrm{MeV}$. As expected, the output nearest the detector is largest. However, it is not possible to compare the contribution of each source cell directly because each source cell has different volume and therefore a different activity.

In fact, MCNP provides a tally in counts per one source photon whether the shape of the source is point or volume. In one case, one photon is released in one disintegration, and one photon is released in one second, then the source activity corresponds to $1 \mathrm{~Bq}$. In this case, the output of the tally means the expected count from a source in $1 \mathrm{~Bq}$.

In order to compare all the source cell data, each volume was multiplied by its output according to the following equation:

$$
\mathrm{Q}(\mathrm{R}, \mathrm{Z})=\mathrm{P}(\mathrm{R}, \mathrm{Z}) \mathrm{V}(\mathrm{R}, \mathrm{Z}) \text {, }
$$

where $Q(R, Z)$ is the normalized pulse count from the $(R, Z)$ cell $[$ count/(photon/ cc)], $P(R, Z)$ is pulse count from the MCNP output from (R,Z) cell [count/photon], and V(R,Z) is the volume of the $(\mathrm{R}, \mathrm{Z})$ cell $[\mathrm{cc}]$. As a result, the normalized count in photon/cc was obtained for each source region. Each value represents the contribution to the count due to that source cell.

\section{Detectable region}

The gamma-rays which can reach the $\mathrm{NaI}$ detector were limited by geometry and self-shielding in the material. A gamma-ray from a point deep in the iron cannot reach the detector due to attenuation. The region where gamma-rays are detectable (the "detectable region") will vary with material and gamma-ray energy.

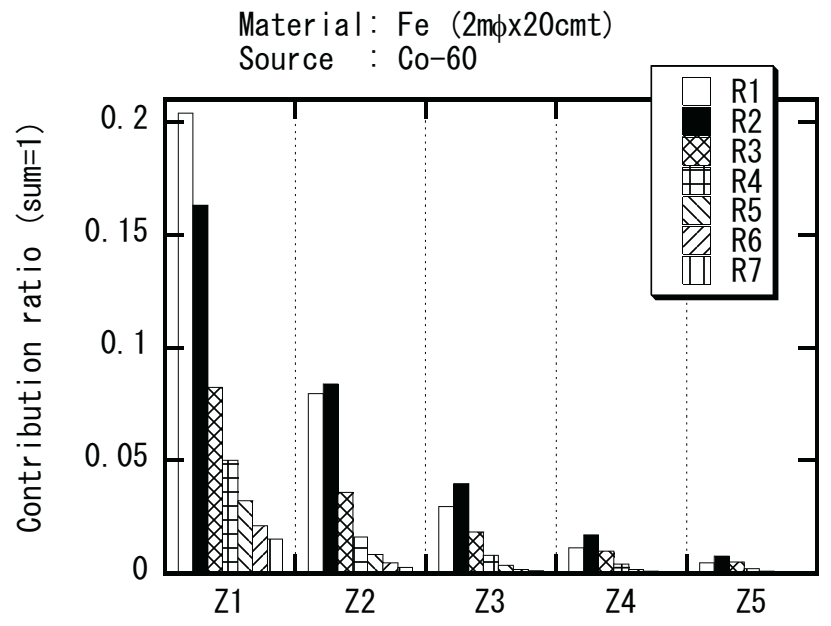

Fig. 2 Contribution ratio of each source cell
Figure 2 shows the contribution ratio of each source cell. The contribution ratio of each $(\mathrm{R}, \mathrm{Z})$ cell is derived from the equation of $\mathrm{Q}(\mathrm{R}, \mathrm{Z}) / \mathrm{Q}$ (Total). Since the value includes volume, some R1 values are less than R2 values on a comparison in the same depth layer.

A detectable region was selected in consideration of each contribution ratio more than 0.025 . The detectable region is shown in Fig. 1 as cells in dark color. The total contribution ratio is $80 \%$.

\section{Discussion of the MDA}

\section{Conversion between MCNP and Experimental data}

The experimental irradiation was done with a $3.44-\mathrm{kBq}$ Co-60 point source at $1 \mathrm{~cm}$ from the surface of the detector. The resulting count rate was 1450 counts/10s after subtracting the background count rate. Therefore, a value of $0.042[\mathrm{cps} / \mathrm{Bq}$ at $1 \mathrm{~cm}]$ was obtained. On the other hand, the MCNP output on the same geometry was calculated as $0.040[\mathrm{cps} / \mathrm{Bq}$ at $1 \mathrm{~cm}]$. As a result, the conversion factor, $\eta$, is 1.05 .

\section{Expected counts from the detectable region with an activity profile}

An activation profile in the induced radioactive materials may be present. The expected counts may be varied with the distribution of activity in the material. Two profile models - the Z-profile and the R-profile, both described below - were assumed for this study's discussion.

The Z-profile model has an activity distribution that changes with depth inside the material. The activity reduces with depth according to an exponential function as the equation (2-1).

$$
\mathrm{A}_{\lambda}(\mathrm{z})=\mathrm{e}^{-\lambda z},
$$

where $A_{\lambda}(z)$ is an activity distribution at the depth $z, \lambda$ is a coefficient for a distribution pattern. Figure 3 shows four patterns of the discrete Z-profile used in this study.

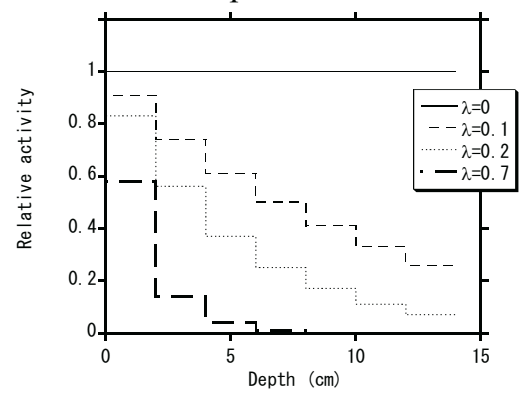

Fig. 3 Z-profile model

The R-profile model has a radial distribution of activity. The activity was assumed to have a Gaussian profile that varied as a function of radius as the equation (2-2).

$$
A_{\sigma}(r)=e^{-r^{2} / 2 \sigma^{2}},
$$

where $A_{\sigma}(r)$ is an activity distribution at the radius $r, \sigma$ is a coefficient for a distribution pattern. Figure 4 shows the four patterns of the discrete R-profile model. 


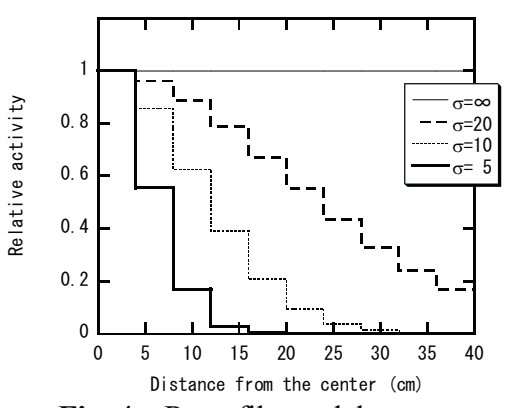

Fig. 4 R-profile model

The expected count of the detector from a unit source cell is derived from the following equations. The equations (3-1) and (3-2) can be applied to the Z-profile model and the R-profile model, respectively.

$$
\begin{aligned}
& C(R, Z)=Q(R, Z) A_{\lambda}(Z), \\
& C(R, Z)=Q(R, Z) A_{\sigma}(R),
\end{aligned}
$$

where $C(R, Z)$ is the expected count from an $(R, Z)$ cell, $\mathrm{Q}(\mathrm{R}, \mathrm{Z})$ is the expected count in an area of uniform activity, $A_{\lambda}(Z)$ and $A_{\sigma}(R)$ is an activity ratio factor from Fig. 3, and Fig. 4. The average specific activity of the detectable region can be calculated from the activity derived from the profile pattern, the volume of the region, and the density of iron:

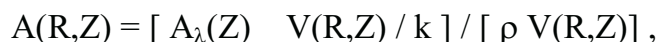

$$
\begin{aligned}
& \mathrm{A}(\mathrm{R}, \mathrm{Z})=\left[\begin{array}{ll}
\mathrm{A}_{\sigma}(\mathrm{R}) & \mathrm{V}(\mathrm{R}, \mathrm{Z}) / \mathrm{k}
\end{array}\right] /[\rho \mathrm{V}(\mathrm{R}, \mathrm{Z})],
\end{aligned}
$$

where $A(R, Z)$ is the specific activity of $(R, Z)$ cell, $k$ is the gamma-ray abundance, and $\rho$ is density of iron. $V(R, Z)$ is the number of photons from the $(\mathrm{R}, \mathrm{Z})$ cell in which the specific activity is in (photon/cc). The equations (4-1) and (4-2) can be applied to the Z-profile model and the R-profile model, respectively. The units of these equations are as follows,

$[\mathrm{Bq} / \mathrm{g}]=[((\mathrm{photon} / \mathrm{cc})(\mathrm{cc})) /($ photon/Bq) $] /[(\mathrm{g} / \mathrm{cc})(\mathrm{cc})]$.

The expected counts in the Ludlum should be obtained using the conversion factor, $\eta$, mentioned in the previous section. Finally, the expected count rate per unit specific activity, S, can be derived by the following equation.

$$
\mathrm{S}=\eta \sum_{\mathrm{R}, \mathrm{Z}} \mathrm{C}(\mathrm{R}, \mathrm{Z}) / \sum_{\mathrm{R}, \mathrm{Z}} \mathrm{A}(\mathrm{R}, \mathrm{Z}) \text {, }
$$

Table 1 shows the expected count rate per unit specific activity, $\mathrm{cpm} /(\mathrm{Bq} / \mathrm{g})$. The sensitivity from this case $(4378$ $\mathrm{cpm} /(\mathrm{Bq} / \mathrm{g}))$ is used in the following discussion concerning MDA.

Table 1 Expected count rate on unit specific activity, $\mathrm{cpm} /(\mathrm{Bq} / \mathrm{g})$, in the detectable region

\begin{tabular}{ccc}
\hline & & $\mathrm{cpm} /(\mathrm{Bq} / \mathrm{g})$ \\
\hline$\lambda=0$ & $\sigma=5$ & 10351 \\
$\lambda=0$ & $\sigma=10$ & 6351 \\
$\lambda=0$ & $\sigma=20$ & 4892 \\
uniform & 4378 \\
$\lambda=0.1$ & $\sigma=\infty$ & 4378 \\
$\lambda=0.2$ & $\sigma=\infty$ & 4405 \\
$\lambda=0.7$ & $\sigma=\infty$ & 4432 \\
\hline
\end{tabular}

\section{MDA.}

The Ludlum Model-18 rate meter will be used for field surveys. The fractional standard deviation on the rate meter is derived as follows, ${ }^{5}$ )

$$
\frac{\sigma_{v}}{v}=\frac{1}{\sqrt{2 \mathrm{yRC}}}
$$

where $v$ is the output on the rate meter, $\sigma_{v} / v$ is fractional standard deviation, $\mathrm{y}$ is count rate, $\mathrm{RC}$ is time constant. There are two response modes for the Model-18: fast which has a time constant of $1.8 \mathrm{~s}$ and slow with a time constant of $10.4 \mathrm{~s}^{2}$. On fast mode a $4-\mathrm{s}$ measuring time is required, and $22 \mathrm{~s}$ is required on slow mode. The count rate in counts per minute $(\mathrm{cpm})$ can be read by the fluctuating needle on the analog scale. The fluctuation width is varied with the time constant and count rate, which can be obtained by using the equation (6). Figure 5 shows the background count rate, the background plus 4 times the standard deviation $\left(4 \sigma_{\mathrm{B}}\right)$ which was derived from the equation (6).

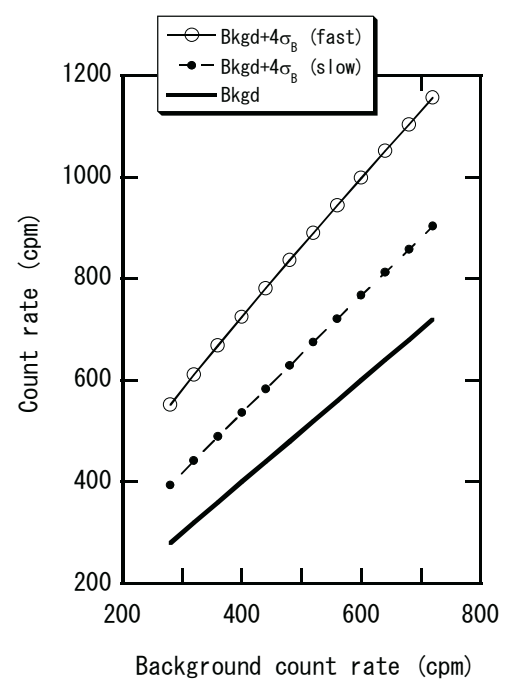

Fig. 5 Background count rate and additional $4 \sigma_{B}$ on using fast and slow response mode

In this study, the minimum detectable activity (MDA) is defined as the count rate corresponding to the $4 \sigma_{\mathrm{B}}$. For the detectable region as shown in Fig. 1, the sensitivity of the Ludlum is $4378 \mathrm{cpm} /(\mathrm{Bq} / \mathrm{g})$ as shown in Table 1, which corresponds to $1 \mathrm{~Bq} / \mathrm{g}$. In one case, the background count rate is $400 \mathrm{cpm}$, then the $4 \sigma_{\mathrm{B}}$ is $136 \mathrm{cpm}$ for the slow response mode. Therefore, $136 \mathrm{cpm}$ corresponds to 0.031 $\mathrm{Bq} / \mathrm{g}$ in consideration with the sensitivity. The value is the MDA using slow response mode on $400 \mathrm{cpm}$ background count rate in this study. In other words, if the background count rate is $400 \mathrm{cpm}$, and the count rate of measuring point is $536 \mathrm{cpm}(=400 \mathrm{cpm}+136 \mathrm{cpm})$, the activity of the detectable region can be estimated to $0.031 \mathrm{~Bq} / \mathrm{g}$ from the net count rate $136 \mathrm{cpm}$ and the sensitivity $4378 \mathrm{cpm} /(\mathrm{Bq} / \mathrm{g})$. Figure 6 shows the MDA using fast and slow response mode. In this case, the target activity of $0.1 \mathrm{~Bq} / \mathrm{g}$ for Co- 60 in iron is detectable. 


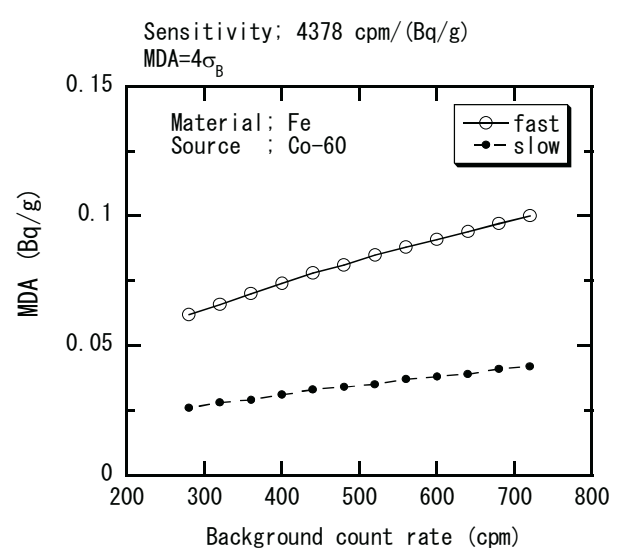

Fig. 6 MDA using fast and slow response mode

\section{R-profile and Off-Center}

In order to examine the decrease in counts when there is some R-profile activity and when the position of the detector is not placed at the profile center, the center of the source was moved horizontally $5 \mathrm{~cm}, 10 \mathrm{~cm}$, and $15 \mathrm{~cm}$ in the MCNP calculation. The source was distributed from the surface of the material to a $20-\mathrm{cm}$ depth; each source cell had a cylindrical shell width of $5 \mathrm{~cm}$; and the maximum radius of each source cell was $40 \mathrm{~cm}$. The pulse count in $\mathrm{NaI}(\mathrm{Tl})$ detector due to each cylindrical-shell-shaped source cell was calculated individually.

Three types of R-profile, $\sigma=20, \sigma=10$ and $\sigma=5$ were assumed as the same as the mentioned above. The expected count was obtained from sum of the count of each cell multiplied by the profile. The larger the distance between the center of the source and the detector becomes, the smaller the count is obtained. Figure 7 shows the trend of the decrease of count ratio with $\mathrm{r} / \sigma$. When $\mathrm{r} / \sigma$ is 1 , the count ratio to $\mathrm{r}=0 \mathrm{~cm}$ is about 0.6 . It is found that even if the distribution has $\sigma=5$ profile, the count is expected more than 0.6 of the peak count on measuring at interval 5 $\mathrm{cm}$. On this condition and the background count rate 720 $\mathrm{cpm}$, the MDA will be derived as $0.07 \mathrm{~Bq} / \mathrm{g}(=0.042$ $[\mathrm{Bq} / \mathrm{g}] / 0.6)$. This $0.042 \mathrm{~Bq} / \mathrm{g}$ is an MDA on $720 \mathrm{cpm}$ from Fig. 6. Therefore, an iron containing Co-60 over $0.1 \mathrm{~Bq} / \mathrm{g}$ can be detected by the survey at interval $5 \mathrm{~cm}$ even when an activity distribution has $\sigma=5$ profile and the background count rate is $720 \mathrm{cpm}$.

\section{Conclusions}

The expected count of the detector was calculated using MCNP. It was set in this calculation that the material for survey is an iron which has $2 \mathrm{~m}$ diameter and $20 \mathrm{~cm}$ thickness, and the radioactive source is Co-60. The calculation model has a R-Z geometry. The detector was set at the center axis and $1 \mathrm{~cm}$ apart from the surface of the material for survey. The unit source cell was set in the material, which has a shape of cylindrical shell with $2 \mathrm{~cm}$ thickness and $4 \mathrm{~cm}$ shell width. The total region of the source was from $0 \mathrm{~cm}$ to $10 \mathrm{~cm}$ in depth, from $0 \mathrm{~cm}$ to $40 \mathrm{~cm}$ in radius. Therefore, total 50 unit source cells were set for

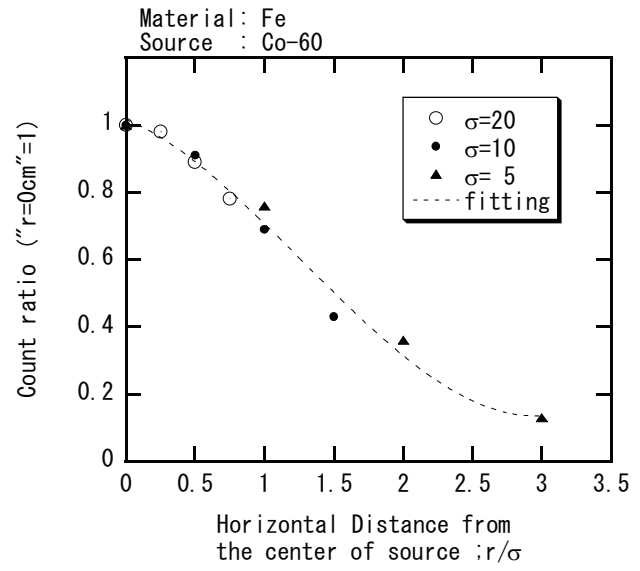

Fig. 7 Trend of decrease of count ratio with $r / \sigma$

the calculation. The specific activity of each cell was assumed as uniform. The expected pulse count was calculated individually for each source cell. As a result, the contribution ratio of each unit source cell to that of total region was obtained from the calculation. It was found that the region which is composed of three layers, $20 \mathrm{~cm}$ radius in $0-2 \mathrm{~cm}$ layer, $12 \mathrm{~cm}$ radius in $2-4 \mathrm{~cm}$ layer and $8 \mathrm{~cm}$ radius in $4-6 \mathrm{~cm}$ layer, has coverage $80 \%$ of contribution. The volume is $3820 \mathrm{cc}$. In other words, the gamma-ray from this region can be detected by the detector.

When the gamma-ray source of Co-60 is distributed in the iron, we can detect it with the sensitivity of $4378 \mathrm{cpm} /(\mathrm{Bq} / \mathrm{g})$ by means of the Ludlum- 18 with a 44-2 detector. The MDA is varied with the background count rate. On the slow response mode of Ludlum-18, if the background count rate is varied from $280 \mathrm{cpm}$ to $720 \mathrm{cpm}$, the MDA is in a range from $0.026 \mathrm{~Bq} / \mathrm{g}$ to $0.041 \mathrm{~Bq} / \mathrm{g}$. As a result, the required detection of $0.1 \mathrm{~Bq} / \mathrm{g}$ can be detected by means of this survey.

The method in this study can be applied to other materials and isotopes, for example, Co-57 in iron or Mn-54 in aluminum.

\section{References}

1) Ludlum Measurements Inc., Manual of Ludlum Model 44-2 Gamma Scintillator, http://www.ludlums.com/manuals/ M44-2_oct07.pdf (2007).

2) Ludlum Measurements Inc., Manual of Ludlum Model 18 Analyzer, $\quad$ http://www.ludlums.com/manuals/M18jun06.pdf (2006).

3) American National Standard, "Surface and Volume Radioactivity Standards for Clearance," ANSI N13.12-1999 (1999).

4) X-5 Monte Carlo Team, MCNP - A General Monte Carlo N-Particle Transport Code Version 5, Volume II: User's Guide, LA-CP-03-0245 (2004).

5) G.F.Knoll, Radiation Detection and Measurement, John Wiley \& Sons, New York, 666 (1979).

6) M.Silari and L.Ulrici, "Investigation of induced radioactivity in the CERN Large Electron Positron collider for its decommissioning," Nucl. Instrum. Methods Phys. Res. A 526, 510 (2004). 